\title{
Heavy Elements and Related New Phenomena
}


This page is intentionally left blank 


\title{
Heavy Elements and Related New Phenomena
}

\author{
Volume I
}

\author{
Editors \\ Walter Greiner \\ Institut für Theoretische Physik \\ Johann Wolfgang Goethe Universität \\ Frankfurt am Main, Germany

\section{Raj K. Gupta} \\ Department of Physics, Panjab University \\ Chandigarh, India
}




\title{
Published by
}

World Scientific Publishing Co. Pte. Ltd.

P O Box 128, Farrer Road, Singapore 912805

USA office: Suite 1B, 1060 Main Street, River Edge, NJ 07661

UK office: 57 Shelton Street, Covent Garden, London WC2H $9 \mathrm{HE}$

\author{
Library of Congress Cataloging-in-Publication Data \\ Heavy elements and related new phenomena / editors, Walter Greiner, \\ Raj K. Gupta. \\ p. $\mathbf{c m}$. \\ Includes index. \\ ISBN 9810233353 (set : alk. paper). -- ISBN 9810239572 (v. 1 :
}

alk. paper), -- ISBN (invalid) $981023958 X$ (v. 2 : alk. paper)

1. Nuclear physics. 2. Superheavy elements. I. Greiner, Walter,

1935- . II. Gupta, Raj K. (Raj Kumar), 1938-

QC770.H4 1999

539.7'234--dc21

99-21157

CIP

\section{British Library Cataloguing-in-Publication Data}

A catalogue record for this book is available from the British Library.

Copyright $\odot 1999$ by World Scientific Publishing Co. Pte. Ltd.

All rights reserved. This book, or parts thereof, may not be reproduced in any form or by any means, electronic or mechanical, including photocopying, recording or any information storage and retrieval system now known or to be invented, without written permission from the Publisher.

For photocopying of material in this volume, please pay a copying fee through the Copyright Clearance Center, Inc., 222 Rosewood Drive, Danvers, MA 01923, USA. In this case permission to photocopy is not required from the publisher. 


\section{PREFACE}

This book consists of four parts, covering both the experimental and theoretical details. The new developments in the field of heavy and superheavy elements and the very related new phenomena of fission and cluster radioactivity are reviewed in the first three Parts I, II and III, respectively. Also, the very new extensions of the Nuclear Chart in new directions of nuclei at the proton and neutron drip-lines, the astrophysical regime and the strange matter are included in Part IV. Some of these considerations could also be of use for future studies of the heavy elements. The contributors were allowed full liberty to give all possible details of the experiments and/or theory so that the book could serve both the post-graduate students in physics as well as the researchers of low energy heavy-ion physics.

Recently, the experiments for the production of heavy/superheavy elements and the related new phenomena have reached a stage of high precision and confidence and the theory, though given out much earlier (two decades or more), is able to explain the underlying physics much better. We have observed not only the "cold fusion" but also the "hot fusion" phenomena, signaling a complete success of the quantum mechanical concept of probability. Similarly, fission is observed both as cold and hot fragments of very high and very low total kinetic energy, respectively. In each case, the cold process is found to be more probable (with larger cross-section) than the hot one, predicted as early as in 1974- 75 by the authors and collaborators. Also, the simultaneous occurance of both cold and hot fission fragments, the bimodal fission, and deformed and/ hyper-deformed fission phenomena are observed and understood on quantum theory. Both the cold fission and cold fusion processes are rare processes since cold fission is masked by fragments of various kinetic energies and cold fusion by their specific choice of target-projectile combinations and incident bombarding energies and angular momenta. Only one or a very few atoms of new heavy elements are thus produced, whose chemistry is now also made possible by the development of new methods. Finally, the very rare process of cluster radioactivity, due to the very large number of competing $\alpha$-decay events, and the availibility of radioactive nuclear beam (RNB) facilities for studying the very neutron-rich or neutron-deficient nuclei at the drip-lines, or the possible nuclear astrophysical processes in nature and the possibility of strange matter (hyper matter) are very interesting new aspects of heavy-ion physics study. The prediction of cluster radioactivity, also as a cold phenomena, once again prior to experiments, establishes beyond doubt the presence of (large amplitude) coherent collective motion, best described by a quantum mechanical theory. This book provides an in-depth study of all these phenomena which are very much related to one another. 
Initially, this book was planned as a single volume of five-, six-hundred pages but the overwhelming response of the contributors has made it grow presently into two volumes. The authors are highly thankful to all the contributors for their valuable contributions and support. Volume I deals with the superheavy elements and fission studies, and Volume II comprises the cluster radioactivity and the very new extensions to regions of drip-line nuclei, the nuclear astrophysics and other new directions. We are concious of the missing one Chapter on experimental details of RNB facilities and their recent new data. Unfortunately, this could not be procured within the time scale set out for the preparation of these two volumes. Lastly, the authors appreciate very much the keen interest and cooperation of the Publishers, particularly for modifying the agreed size of the book as 8.5 inches by 6 inches to an impressive 9.75 inches by 6.5 inches.

Walter Greiner

Raj K. Gupta 


\section{CONTENTS}

\section{Volume 1}

Preface V V

General Introduction 3

Part I New Developments in the Study of Superheavy Elements: Experiments and Theory

Chapter 1 Discovery of the Heaviest Elements

G. Münzenberg and S. Hofmann

Chapter 2 Present and Future Prospects for Synthesis of Heavy Nuclei

Yu.Ts. Oganessian

Chapter 3 Cold Multinucleon Transfer and Synthesis of New Elements

W. von Oertzen

Chapter 4 Chemical Properties of the Transactinide Elements J.V. Kratz

Chapter 5 Electronic Structure and Chemistry of the Heaviest Elements

V. Pershina and B. Fricke

Chapter 6 Valence Correlation Schemes and the Structure of Heavy Actinide Nuclei

R.F. Casten and N.V. Zamfir

Chapter 7 Skyrme-Hartree-Fock Theory for Shell Structure of Superheavy Elements

S. Ćwiok and P. Magierski

Chapter 8 Superheavy Nuclei in Deformed Mean-field Calculations P.-G. Reinhard and J.A. Maruhn

Chapter 9 New Islands of Stability Using the Finite-potential Approach to Superheavy Elements

A. Sobiczewski 
viii Contents

Chapter 10 Two-centre Shell Model in Cold Synthesis of Superheavy Elements

R.K. Gupta and W. Greiner

Part II New Developments in Fission Physics:

Experiments and Theory

Chapter 11 Cold Fission

H.-G. Clerc

Chapter 12 High Neutron Multiplicity and Cold Binary and Ternary Spontaneous Fission of ${ }^{252} \mathrm{Cf}$

A.V. Ramayya, J.H. Hamilton, J.K. Hwang and G.M. Ter-Akopian

Chapter 13 Bimodal Nature of Nuclear Fission

507

T. Ohtsuki, Y. Nagame and H. Nakahara

Chapter 14 Quantum Mechanical Fragmentation Theory for Cold Distribution of Masses and Charges in Fissioning Nuclei and Nuclei Formed in Heavy Ion Reactions

R.K. Gupta and W. Greiner

Chapter 15 Fission through Quasi-molecular Shapes and

Fragmentation

591

G. Royer

Author Index

\section{Volume 2}

Preface

General Introduction

637

Part III New Cluster Radioactivity and the Superasymmetric Fission: Experiments and Theory

Chapter 16 Measurements on Cluster Radioactivity - Present Experimental Status

R. Bonetti and A. Guglielmetti

Chapter 17 Numerical and Analytical Super-asymmetric Fission Model for Exotic Cluster Decays 
Chapter 18 Collective Description of Exotic Cluster Decays and Shell Structure Effects of Parent/Daughter Nuclei R.K. Gupta

Chapter 19 Fine Structure in Cluster Radioactivity 765 M. Mirea and R.K. Gupta

Chapter 20 Super-asymmetric Cold Fission and Exotic

Cluster-decay Processes R.K. Gupta and W. Scheid

Chapter 21 Cold Binary and Ternary Fragmentations as an Extension of Cluster Radioactivity

A. Săndulescu, F. Cărstoiu, S. Misicu, A. Florescu,

I. Bulboacă, A.V. Ramayya, J.H. Hamilton, J.K. Hwang, G.M. Ter-Akopian and W. Greiner

Part IV Extensions in New Directions: Nuclear Astrophysics, Physics of Nuclei Near Drip-lines and Strange Matter: Experiments and Theory

Chapter 22 Nuclear Astrophysics at the Beginning of the Twenty-first Century R.N. Boyd

Chapter 23 Two- and Three-body Properties of Halo Nuclei 976 I.J. Thompson and J.S. Vaagen

Chapter 24 Properties of Light Nuclei Near Drip-lines in the Relativistic Mean-field Theory S.K. Patra, R.K. Gupta and W. Greiner

Chapter 25 Heavy-ion Fusion Reactions at Energies Below the Couloumb Barrier N. Takigawa and K. Hagino

Chapter 26 Neutron Drip-line Nuclei: Their Halo Structure, Synthesis, and Decay via Cluster Emissions R.K. Gupta, R.K. Puri, W. Scheid and W. Greiner

Chapter 27 Physics of Strange Matter Carsten Greiner and J. Schaffner-Bielich 